\title{
ANÁLISE COMPARATIVA DA ASPERSÃO DE CARBETO DE TUNGSTÊNIO EM TITÂNIO PELOS PROCESSOS DE HVOF E PLASMA SPRAY
}

\author{
Franciane Galvão Faria ' \\ Mariana Anastácia de Oliveira Schwarc ' \\ Claudinei Milan Tavares ' \\ Leandro Guimarães de Oliveira ${ }^{2}$ \\ Jose Cristiano Pereira'
}

\section{Resumo}

O objetivo deste estudo é verificar e comparar a qualidade do revestimento de carbeto de tungstênio - cobalto ( $83 \%$ WC e 17\% Co) aplicado sobre material base de titânio por Plasma Spray e HVOF (High Velocity Oxygen Fuel), realizando análises de microestrutura para verificar o percentual de porosidade, espessura, tração e dureza, em amostras. Este estudo é de grande importância por haver um crescente uso desse revestimento para fornecer resistência ao desgaste com altos níveis de dureza e tenacidade, às superfícies de componentes, em muitas áreas da indústria. $O$ material base escolhido para o estudo é a liga de titânio Ti6Al-4V (norma SAE AMS 49II). A análise das amostras apresentou que ambos os processos foram satisfatórios, com uma pequena vantagem no processo HVOF que produz revestimentos mais densos, duros e mais resistentes, além de ser uma boa escolha por suas características, considerando os requisitos de alta qualidade em muitas áreas das indústrias, como restauração de peças usadas na área de aviação. A análise apresentada aqui pode ser uma fonte inestimável de informações para profissionais e engenheiros de manufatura, no sentido de enriquecer seu conhecimento sobre esse tipo de revestimento e facilitar a decisão sobre quais processos utilizar.

Palavras-chave: Aspersão térmica; Plasma Spray; HVOF; Carbeto de tungstênio.

\section{COMPARATIVE ANALYSIS OF TUNGSTEN CARBIDE THERMAL SPRAY IN TITANIUM BY HVOF AND PLASMA SPRAY PROCESSES}

\begin{abstract}
The objective of this study is to verify and qualitatively compare the mechanical properties of tungsten - cobalt carbide deposition by Plasma Spray or High Velocity Oxygen Fuel (HVOF) processes on titanium base material, using tests such as metallography, traction and hardness. This study is of great importance due to the increasing use of this coating to improve wear resistance and surface hardness of components in various industry areas. The base material chosen for this study is titanium Ti6Al4V grade 5 (according to SAE 49I I), an alloy widely used for its characteristics such as weight and durability. The analysis of the samples shows that both spraying processes were satisfactory with a slight advantage of the HVOF process which produces a harder and denser coating and is a good choice if you consider the high restoration quality requirements of surface area parts. aviation, for example. This analysis presented here can be an invaluable source of information for manufacturing professionals and engineers to enrich their knowledge of this type of coating and make it easier to decide which processes to use.
\end{abstract}

Keywords: Thermal spray; Plasma Spray; HVOF; Tungsten carbide.

\section{INTRODUÇÃO}

O objetivo desse estudo é avaliar, para os processos de Thermal Spray, Plasma Spray e HVOF, a aplicação de carbeto de tungstênio- cobalto $(83 \%$ WC e $17 \% \mathrm{Co})$ em material base de titânio. Esta análise é de grande importância uma vez que há uma crescente utilização, pela indústria, de processos de reparo ou modificação de características de

'Universidade Católica de Petrópolis - UCP, Petrópolis, RJ, Brasil. E-mail: ane.gf2@gmail.com

2Instituto SENAI de Tecnologia em Solda, Rio de Janeiro, RJ, Brasil.

2176-1523 (C) 2020 Associação Brasileira de Metalurgia, Materiais e Mineração. Publicado pela ABM. Este é um artigo de acesso aberto distribuído sob os termos da licença Creative Commons CC BY-NC-ND (Attribution-NonCommercial-NoDerivs) - https:// creativecommons.org/licenses/by-nc-nd/4.0\%. 
produtos, devido aos elevados custos de fabricação, falta de matéria prima, entre outros problemas.

O uso de revestimentos duros para melhorar a resistência ao desgaste de componentes mecânicos tem sido comum há várias décadas, com variadas técnicas de aplicação, sendo a aspersão térmica a técnica que mais tem se desenvolvido nos últimos anos, apresentando-se como alternativa, economicamente viável, em relação aos demais processos comumente utilizados $[1,2]$.

Eles podem ser aplicados por processo de aspersão térmica como Plasma Spray, HVOF ou Detonation Gun [3]. A versatilidade do processo é aprimorada ainda mais pela ampla gama de materiais que podem ser pulverizados e pela possibilidade de revestir peças com diferentes dimensões e geometrias [4]. Além disso, esses materiais vêm sendo especificados para aplicações críticas em componentes de aeronaves, como trêns de pouso. Os revestimentos de superfície WC-Co são comumente utilizados para aumentar a resistência ao desgaste de muitos tipos de componentes, depositados via Plasma Spray (Air Plasma Spray) e HVOF [5].

Estudos anteriores apresentam resultados como a influência do cobalto no processo de deposição por HVOF, na resistência à erosão por cavitação dos revestimentos WC-Co [4] e a investigação do comportamento do desgaste do revestimento carbeto em HVOF [6] ou a resistência ao desgaste abrasivo do tungstênio aspergido por plasma [7] e comparação entre pós usados no plasma e HVOF em carbeto de tungstênio com uma proporção diferente de cobalto [5]. Nenhum dos estudos anteriores acima abordou a comparação do Plasma Spray (APS) (Air Plasma Spray) com HVOF comumente usado para esse tipo de aplicação, com um material específico e substrato de titânio.

Este estudo é muito importante para muitas áreas da indústria, como aeroespacial. Atualmente, alguns aviões comerciais, como o AirBus A380 e o Boeing B787, usam muito mais titânio do que as aeronaves previamente projetadas [8], devido à sua alta relação entre força e peso e resistência à corrosão. Tribologicamente, as superfícies de Ti6Al4V são especialmente suscetíveis à fricção, produzindo resíduos de óxido duro que ficam na interface [9].

Este estudo mostra os resultados das análises de microestrutura, percentual de porosidade, tração, espessura e teste de dureza de ambos os processos, nas amostras selecionadas. A contribuição é significativa, pois permite que os tomadores de decisão escolham qual é o método mais adequado para restaurar, por exemplo, componentes de engenharia com propriedades de alto desempenho que operam com altos requisitos de superfície, como propriedades tribológicas.

Acredita-se que o presente estudo amplie o conhecimento de profissionais como gerentes, engenheiros de projeto, reparo e fabricação que precisam especificar métodos de pulverização de componentes que exijam proteção contra desgaste, altos níveis de dureza para proteger materiais de titânio.
Este artigo fornece respostas para perguntas de pesquisa como: Qual é a diferença de qualidade entre o processo de Plasma Spray e o processo HVOF? E qual é a principal vantagem do uso do HVOF em vez do processo de Plasma Spray?

O artigo está estruturado da seguinte forma: a Seção 2 está focada em apresentar os materiais e métodos deste estudo. A seção 3 apresenta os resultados e discussão, a seção 4, a conclusão de todas as análises e resultados e, ao final, as referências verificadas para enriquecer este estudo.

\section{MATERIAIS E MÉTODO}

O material selecionado para o substrato foi o de titânio Ti6Al4V grau 5 (conforme norma SAE 49I I) [8], devido a sua aplicabilidade na indústria aeronáutica. Os corpos de prova foram selecionados em conformidade com as boas práticas de qualificação de processo, utilizadas na área de aviação. Para cada processo, Plasma e HVOF, serão utilizados uma chapa e três buttons. As dimensões da chapa retangular foi de 50,8 $\times 25,4 \mathrm{~mm}(2 \times \mathrm{I}$ in), e espessura de I mm (0,040 in). Os buttons circulares utilizados na tração, possuem diâmetro de $25,4 \mathrm{~mm}$ ( $\mathrm{I}$ in) e espessura de 5 , I mm ( 0,2 polegada).

O Material do revestimento utilizado consiste em uma composição de $83 \%$ de carbeto de tungstênio, como material duro, e uma matriz de $17 \%$ de cobalto, que funciona como um material aglutinante para as partículas de carbeto. Estes produtos são esferoidais, aglomerados e sinterizados para aspersão térmica. Este revestimento, comparado com outros de menor proporção de cobalto, apresenta maior resistência mecânica e resistência ao desgaste, como resultado de maiores níveis de cobalto. Já o carbeto de tungstênio protege o substrato dos efeitos da fricção, grãos abrasivos, erosão de partículas, desgaste deslizante, desgaste de martelo (resistência ao impacto), cavitação e contato dinâmico com superfícies duras de acordo com o fabricante [10].

O primeiro passo para iniciar o processo de aspersão é a preparação da superfície que consiste em limpeza com desengraxante, em seguida passam pelo processo de shot peen (jateamento por partículas esféricas) com material de jateamento CCWI4 a 207 kPa (30 PSI) de pressão. Por fim elas receberam jateamento abrasivo na face que irá receber o revestimento com pressão que varia de 344-4I4 kPa (50-60 PSI).

Realizou-se o procedimento de aspersão por dois métodos, HVOF, equipamento Jet Kote III e Plasma Spray, 9MC Sulzer Metco, para comparação das aplicações. Os parâmetros foram pré-estabelecidos conforme manual do fabricante e atende a requisitos da área de aviação.

Para o ensaio de microdureza Vickers, foi utilizada carga de $300 \mathrm{~g}$ realizando com 10 leituras distribuídas ao longo da área de análise. Já para o ensaio de macrodureza, a escala utilizada é a Rockwell I5N, que de acordo com a 
norma ASTM E-I8 [I I] indica a utilização de penetrador de diamante, pré-carga de $3 \mathrm{kgf}$ e carga de $15 \mathrm{kgf}$.

A caracterização da resistência à tração dos dois processos utilizou ensaio de aderência conforme norma ASTM C-633 [12]. O ensaio resume-se na colagem (com adesivo poliamida-epóxi que seja viscoso o suficiente para não penetrar $0,38 \mathrm{~mm}(0.015 \mathrm{in})$ ) de dois "corpos de prova de extração" sem revestimento, um em cada lado da superfície plana do button revestido. Após a cura e esfriamento o conjunto é tracionado a uma taxa de $1.016 \mathrm{~mm} / \mathrm{min}(0.040 \mathrm{in} / \mathrm{min})$ em uma máquina de ensaio de tração. A resistência à tração é a razão entre força máxima e a área de ruptura.

As avaliações metalográficas, de forma geral, buscam quantificar o percentual de porosidade, medir, de forma mais precisa, a espessura, verificar a presença de trincas, delaminações e grau de oxidação. As chapas foram cortadas com disco abrasivo. Posteriormente os pedaços foram embutidos em resina epoxy-mineral de cura a quente. A superfície para análise micrográfica das amostras foi preparada, utilizando-se lixas de carbeto de silício com granas $180,220,320,400,600$ e 1200 sob refrigeração a água. Por fim, as amostras passaram por polimento mecânico com diamante de $1 \mu \mathrm{m}$ e depois por sílica coloidal de $0,04 \mu \mathrm{m}$.

Para os testes de macrodureza, é estipulado, de acordo com a norma internacional ASTM E-I8 [II], uma espessura mínima de $0,357 \mathrm{~mm}(0,014 \mathrm{in})$. Além disso, sabe-se que esta espessura máxima é segura para a aderência do revestimento. Já para os corpos de prova de tração, a norma ASTM C-633 [12], estipula uma espessura mínima de $0,381 \mathrm{~mm}$ ( 0.015 in) medido por micrômetro. A medição da espessura na metalografia foi realizada de forma distribuída entre toda a amostra, foram medidos, um pico, depois um vale, nessa ordem, até obter-se cinco leituras de picos e cinco leituras de vale (Figura I). Calcula-se a média desses valores, e aí então tem-se a espessura média do revestimento na chapa. Já a espessura do button é baseada apenas na medição feita pelo operador com micrômetro de ponta plana. A ampliação é um fator importante, sendo considerado ideal para medição de espessura, uma ampliação de 100 a 200 vezes.

Para realização do cálculo do percentual de porosidade existente das amostras, as micrografias foram avaliadas por um software analisador de imagem (image $j$ ). $O$ procedimento segue a norma ASTM E-2109 [13]. Um quadro é escolhido e em seguida faz-se a seleção dos poros no campo de visão. Isto consiste em selecionar a gama apropriada que preencha a maior parte dos poros. Foram avaliados três quadros aleatórios de cada amostra para realização do percentual de poro.

\section{RESULTADOS E DISCUSSÃO}

Foi realizada análise em Microscópio Eletrônico de Varredura ou MEV para obter mais detalhes sobre a variação da superfície das amostras de titânio.

$\mathrm{Na}$ Figura 2 verifica-se o efeito "martelamento" do processo de shot peen, originando na superfície uma pequena microcavidade por escoamento das camadas superficiais.

Essa microcavidade foi criada devido a tensão gerada pelo processo ser maior que o limite de escoamento do material da superfície. Abaixo da superfície essas camadas tendem a reestabelecer a forma original.

$\mathrm{Na}$ Figura 3 foi analisada a seção transversal da amostra após o shot peen, com ampliação de 3000x, onde pode-se perceber a variação na organização da microestrutura próxima a superfície. As setas indicam o sentido dessa organização.

As setas negras representam a deformação da microestrutura na superfície, e as setas brancas, a organização da mesma, em camadas mais profundas que não foram aparentemente atingidas pela deformação criada pelo processo

Por fim, o jateamento abrasivo vem fornecer a rugosidade necessária para ancoramento das partículas aspergidas, veja Figura 4. A rugosidade do início do processo até esta etapa aumentou 4 vezes chegando a $100 \mathrm{Ra} \pm 15 \mathrm{Ra}$.

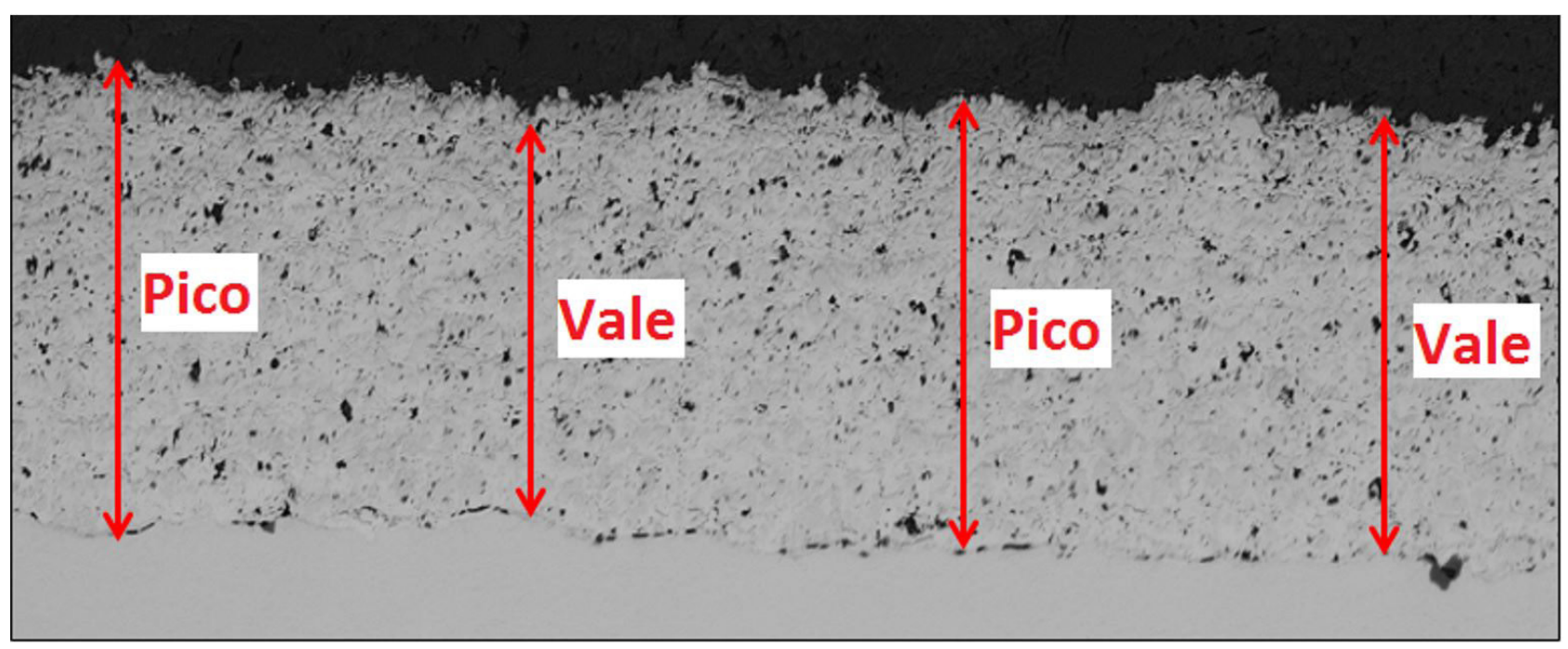

Figura I. Método de medição de espessura. 

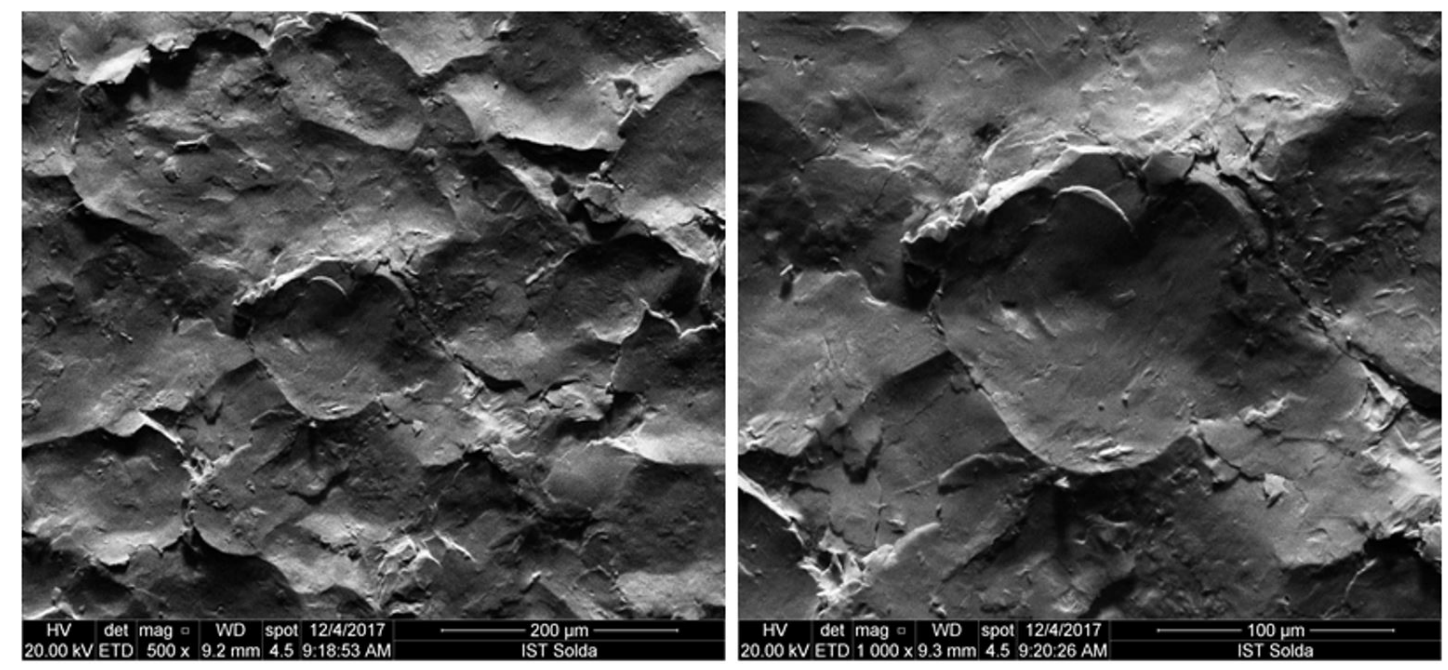

Figura 2. Foto em MEV da amostra de tração após o processo de shot peen.

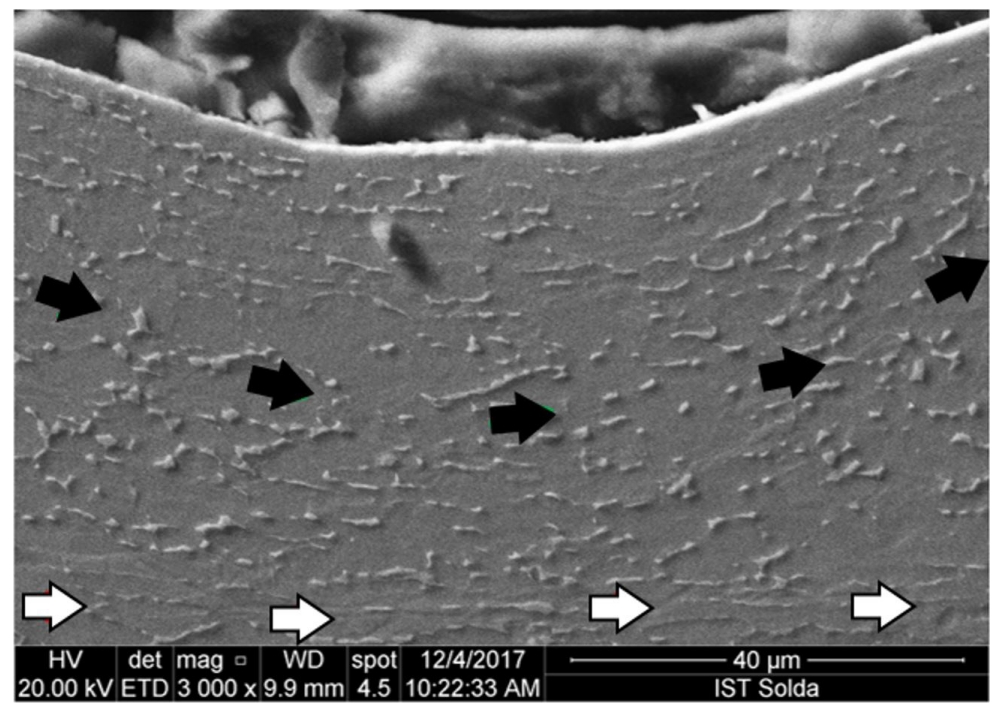

Figura 3. Imagem da seção transversal da amostra após o shot peen.
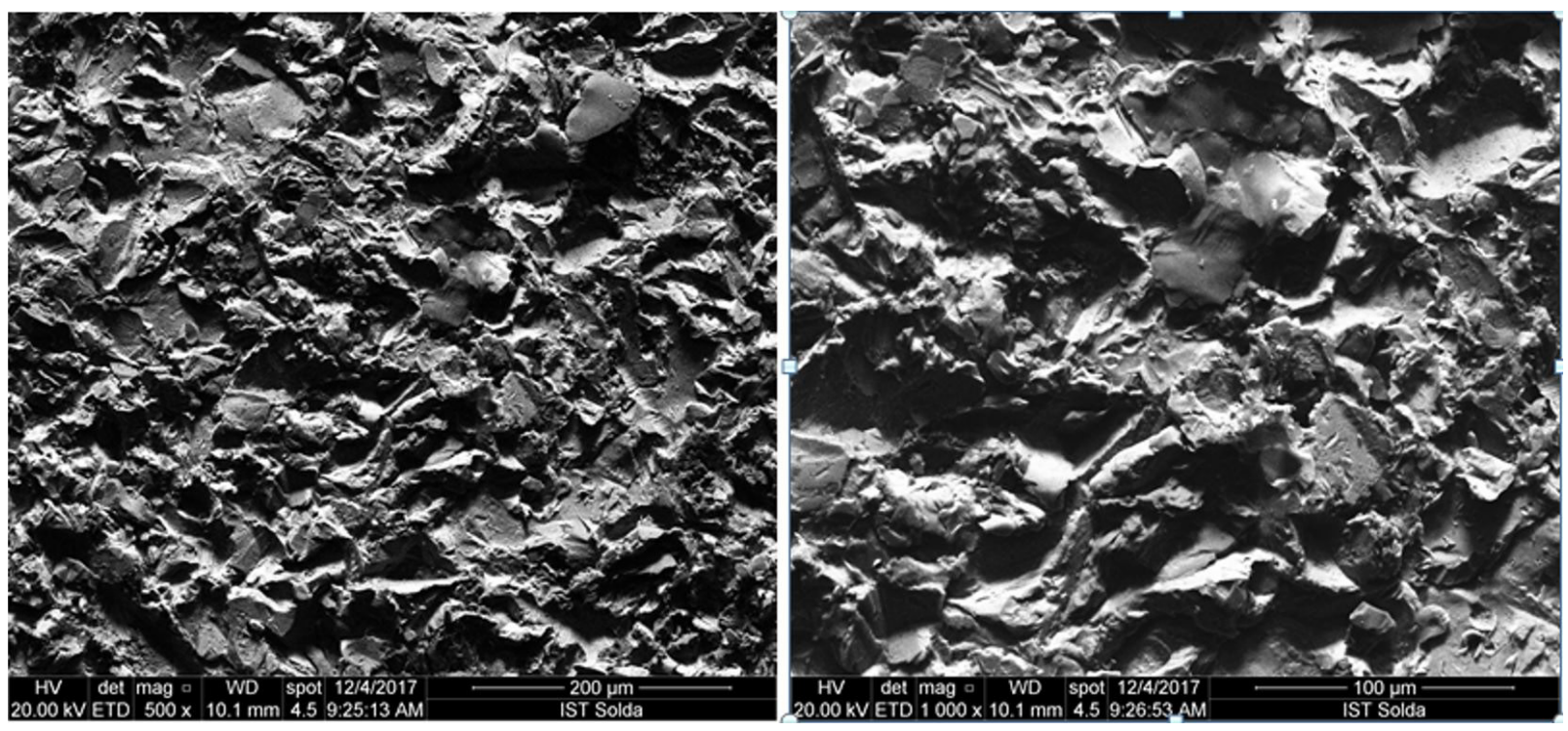

Figura 4. Foto em MEV da superfície em seu estado final, após o shot peen e o jateamento abrasivo. 
Pode-se avaliar a microestrutura do revestimento de carbeto de tungstênio - Cobalto, como a seguir na Figura 5, para cada processo estudado com ampliação de 500x.

A microestrutura do carbeto de tungstênio é caracterizada basicamente por duas fases, a escura ou “dark fase”, que é o material carbeto (representado na imagem pela parte cinza escuro) e a fase clara ou "white fase" (representada pela parte cinza claro) que constitui o cobalto. Essas duas fases devem estar bem distribuídas para garantir a qualidade do processo. Pode-se perceber que para o processo de HVOF as partículas são menores que no de Plasma. Isso pode ser explicado pela alta velocidade em que as partículas são lançadas contra o substrato. Não houve presença de trincas, delaminações ou qualquer outro problema de aplicação em ambas às amostras.

A espessura média, feita por microscópio do processo de plasma foi de $0,356 \pm 0,001 \mathrm{~mm}(14 \pm 0.04 \mathrm{mil})$ e para HVOF 0,38I $\pm 0,001 \mathrm{~mm}$ ( $15 \pm 0.04 \mathrm{mil})$. Verificou-se maior variação de espessura no revestimento aplicado pelo processo de Plasma Spray do que no de HVOF. Isso se dá pela alta velocidade em que as partículas são aspergidas neste último, fazendo com que o revestimento se forme de forma mais "lisa" e compacta (Figura 6). Além disso, o processo de HVOF tem a característica de apresentar baixas tensões residuais trativas ou compressivas, o que permite aplicar maiores espessuras.

Já a medição com micrômetro de ponta plana foi de $0,432 \mathrm{~mm} \pm 0,007 \mathrm{~mm}$ (I7 mil $\pm 0.028 \mathrm{mil})$ para $\circ$ HVOF e 0,432 mm $\pm 0,007 \mathrm{~mm}$ ( $17 \mathrm{mil} \pm 0.028 \mathrm{mil})$, para o Plasma. Essa medição foi maior se comparada com a realizada metalograficamente pois a ponta do micrometro fica limitada aos picos do revestimento. Quanto mais “lisa” a superfície, mais precisa será a medição por este instrumento.

A análise da porosidade foi realizada por software (Image $J$ ) em três quadros de cada amostra, e apresentou os seguintes resultados (Tabela I e Tabela 2):

Pode-se perceber a elevação do percentual de porosidade (quase 4 vezes mais) e do tamanho de poros (aproximadamente o dobro do tamanho), encontrado no processo de Plasma Spray, comparado com o processo de HVOF. Isto é característico do HVOF, devido à alta velocidade em que as partículas atingem o substrato e a baixa temperatura de aspersão. Como o processo de Plasma Spray é realizado a temperaturas mais elevadas (aproximadamente 6 vezes mais), a partícula dilata mais, e após atingir o substrato tende a
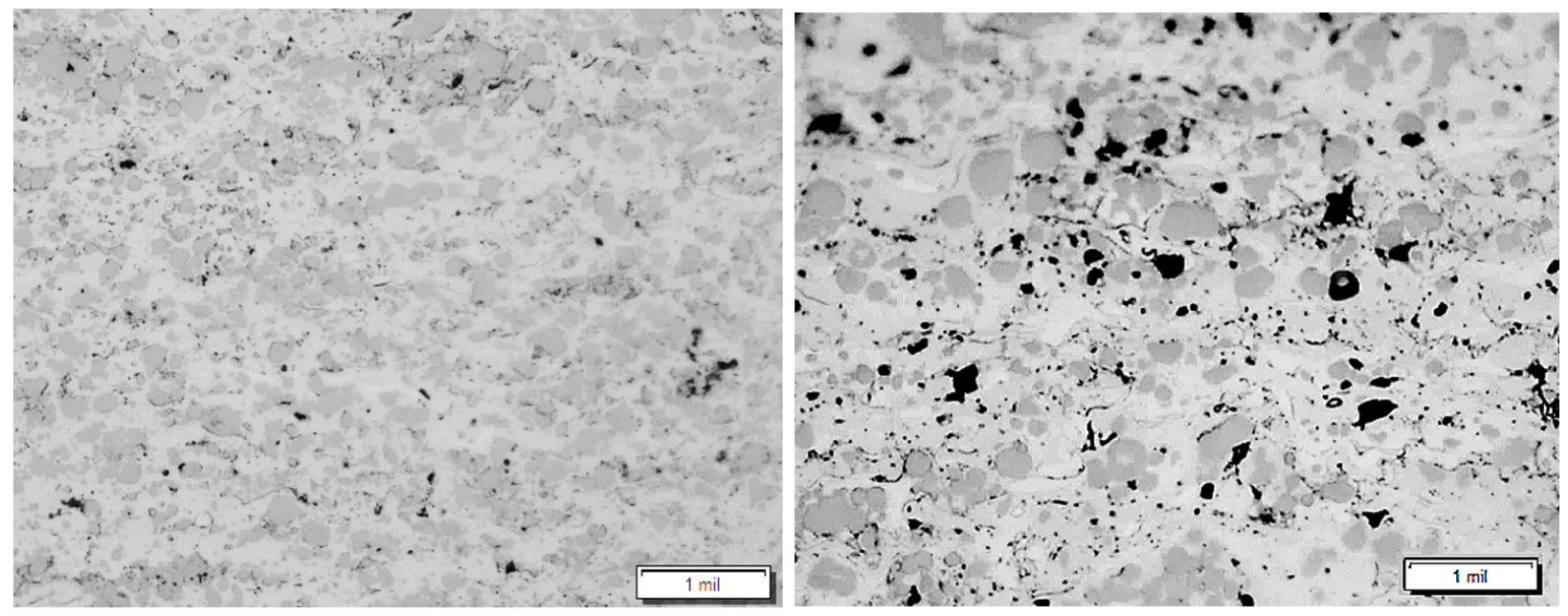

Figura 5. Microestrutura Carbeto de Tungstênio - HVOF a esquerda e Plasma a direita.

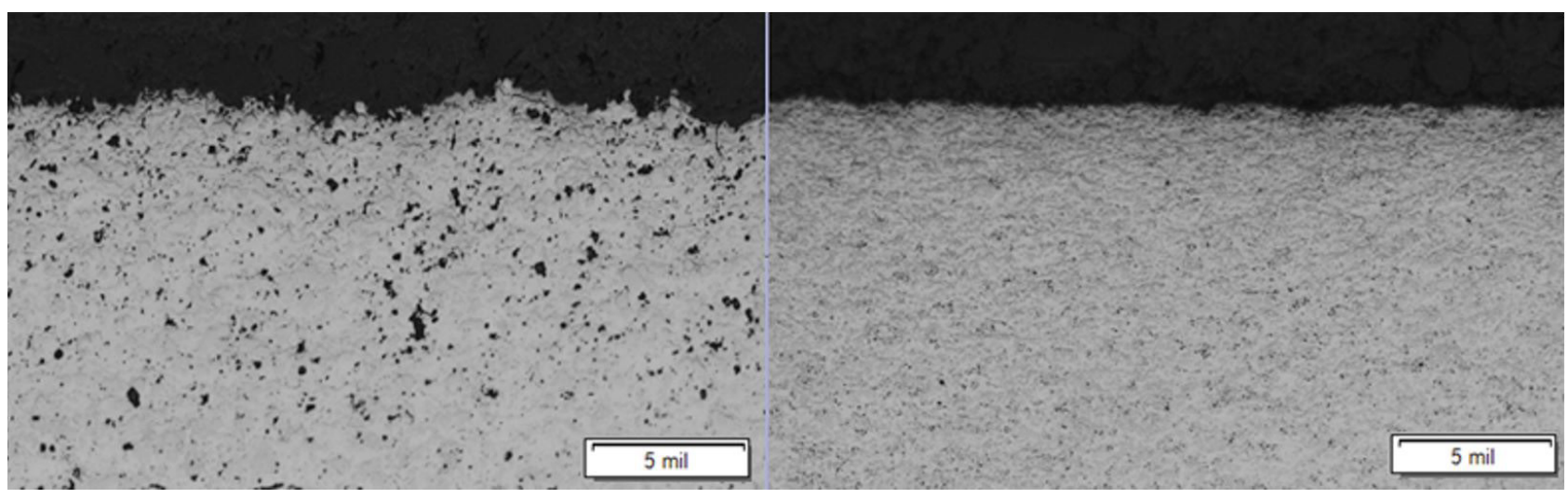

Figura 6. Foto comparativa da variação nas superfícies (a esquerda do processo de Plasma e a direita do processo de HVOF). Fonte: Elaborado pela autora. 
comprimir bastante, deixando um espaço entre uma partícula e outra, formando-se assim o poro.

A variação de microdureza e macrodureza está apresentada nas Tabelas 3 e 4 acima, para cada processo. Ao analisar-se os resultados obtidos, pode-se verificar que a aspersão pelo processo de HVOF apresenta maiores resultados de dureza, possivelmente pela maior coesão entre as partículas (poucos vazios entre elas), revestimento mais denso, se comparado com o processo de Plasma.

Os resultados dos testes de tração para cada processo em estudo são apresentados na Tabela 5.

A Figura 7 a seguir mostra o tipo de ruptura, que foi encontrada para os três corpos de prova, de ambos os processos, a direita para aspersão por HVOF e a esquerda, por Plasma Spray.

Ambos testes de aderência tiveram valores semelhantes, porém o de HVOF resistiu um pouco mais, rompendo na cola, nos três corpos de prova. Essa conclusão é alcançada verificando-se que a parte branca (cola) é predominante e o revestimento aparece praticamente intacto. Em contrapartida, nos corpos de prova do processo de Plasma, verificamos que no segundo corpo de prova, em ambos os lados têm-se revestimento, isso significa que o revestimento foi mais frágil. Outra importante informação é perceber ambos os processos atingiram a espessura mínima requerida pela norma ASTM C-633 [12], que exigi um valor mínimo de 0.0 I $5 \mathrm{in}$.

Tabela I. Análise de porosidade Plasma Spray

\begin{tabular}{|c|c|c|c|c|c|}
\hline \multicolumn{6}{|c|}{ ANÁLISE DE POROSIDADE - Plasma Spray } \\
\hline & Quadro I & Quadro 2 & Quadro 3 & Média & Desvio Padrão \\
\hline $\begin{array}{l}\text { Percentual de } \\
\text { Porosidade }\end{array}$ & 2,04 & 2,87 & 1,65 & 2,19 & 0,62 \\
\hline Tamanho Máximo & 22,81 & 23,29 & 31,19 & $25,76 \pm 0,005 \mu \mathrm{m}$ & $4,70 \pm 0,005 \mu \mathrm{m}$ \\
\hline$\mu \mathrm{m}$ & $(0,90)$ & $(0,91)$ & $(1,20)$ & $(I, 0 \mathrm{I} \pm 0,0002 \mathrm{mil})$ & $(0,0002 \pm 0,0002 \mathrm{mil})$ \\
\hline (mil) & & & & & \\
\hline
\end{tabular}

Tabela 2. Análise de porosidade HVOF

\begin{tabular}{|c|c|c|c|c|c|}
\hline \multicolumn{6}{|c|}{ ANÁLISE DE POROSIDADE - HVOF } \\
\hline & Quadro I & Quadro 2 & Quadro 3 & Média & Desvio Padrão \\
\hline Percentual de Porosidade & 0,64 & 0,80 & 0,53 & 0,66 & 0,13 \\
\hline Tamanho Máximo & 10,60 & 18,29 & 13,16 & $14,02 \pm 0,005 \mu \mathrm{m}$ & $3,91 \pm 0,005 \mu \mathrm{m}$ \\
\hline$\mu \mathrm{m}$ & $(0,42)$ & $(0,72)$ & $(0,52)$ & $(0,55 \pm 0,0002 \mathrm{mil})$ & $(0,15 \pm 0,0002 \mathrm{mil})$ \\
\hline (mil) & & & & & \\
\hline
\end{tabular}

Tabela 3. Valores de microdureza (Vickers)

\begin{tabular}{|c|c|c|c|c|c|c|c|c|c|c|c|c|}
\hline \multicolumn{13}{|c|}{ Microdureza Vickers } \\
\hline Processo & \multicolumn{10}{|c|}{ Leituras [HV 0.3] } & \multirow{2}{*}{$\begin{array}{c}\text { Média } \\
981\end{array}$} & \multirow{2}{*}{$\begin{array}{c}\begin{array}{c}\text { Desvio } \\
\text { Pedrão }\end{array} \\
\text { [HV 0,3] } \\
8,92\end{array}$} \\
\hline HVOF & 981 & 981 & 970 & 989 & 978 & 994 & 973 & 987 & 979 & 968 & & \\
\hline PLASMA SPRAY & 917 & 917 & 925 & 898 & 930 & 903 & 916 & 932 & 927 & 917 & 917 & 12,20 \\
\hline
\end{tabular}

Tabela 4. Valores de macrodureza (Rockwell)

\begin{tabular}{|c|c|c|c|c|c|c|c|}
\hline \multicolumn{8}{|c|}{ Dureza Rockwell } \\
\hline Processo & & & as [ & & & Média & Desvio Padrão \\
\hline HVOF & 92 & 92 & 91 & 93 & 92 & $92 \mathrm{HRI} 5 \mathrm{~N}$ & $0,7 \mathrm{HRI} 5 \mathrm{~N}$ \\
\hline PLASMA SPRAY & 88 & 88 & 89 & 88 & 89 & $88 \mathrm{HRI} 5 \mathrm{~N}$ & $0,55 \mathrm{HRI} 5 \mathrm{~N}$ \\
\hline
\end{tabular}

Tabela 5. Valores encontrados no ensaio de tração

\begin{tabular}{ccccccc}
\hline & \multicolumn{3}{c}{ Resistência à Tração } \\
\hline & $\begin{array}{c}\mathbf{I}^{\circ} \text { Corpo de } \\
\text { Prova }\end{array}$ & $\begin{array}{c}\mathbf{2}^{\circ} \text { Corpo de } \\
\text { Prova }\end{array}$ & $\begin{array}{c}\mathbf{3}^{\circ} \text { Corpo de } \\
\text { Prova }\end{array}$ & Média & Máximo & Desvio Padrão \\
\hline Plasma Spray & $47 \mathrm{Mpa}$ & $5 \mathrm{IMpa}$ & $54 \mathrm{Mpa}$ & $5 \mathrm{I} \mathrm{Mpa}$ & $54 \mathrm{Mpa}$ & $3,7 \mathrm{Mpa}(536 \mathrm{PSI})$ \\
& $(6795 \mathrm{PSI})$ & $(7439 \mathrm{PSI})$ & $(7860 \mathrm{PSI})$ & $(7365 \mathrm{PSI})$ & $(7860 \mathrm{PSI})$ & \\
HVOF & $55 \mathrm{Mpa}$ & $59 \mathrm{MPa}$ & $56 \mathrm{Mpa}$ & $56 \mathrm{Mpa}$ & $59 \mathrm{Mpa}(853 \mathrm{IPSI})$ & $2, \mathrm{I} \mathrm{Mpa}(302 \mathrm{PSI})$ \\
& $(7948 \mathrm{PSI})$ & $(853 \mathrm{IPSI})$ & $(8099 \mathrm{PSI})$ & $(8192 \mathrm{PSI})$ & & \\
\hline
\end{tabular}




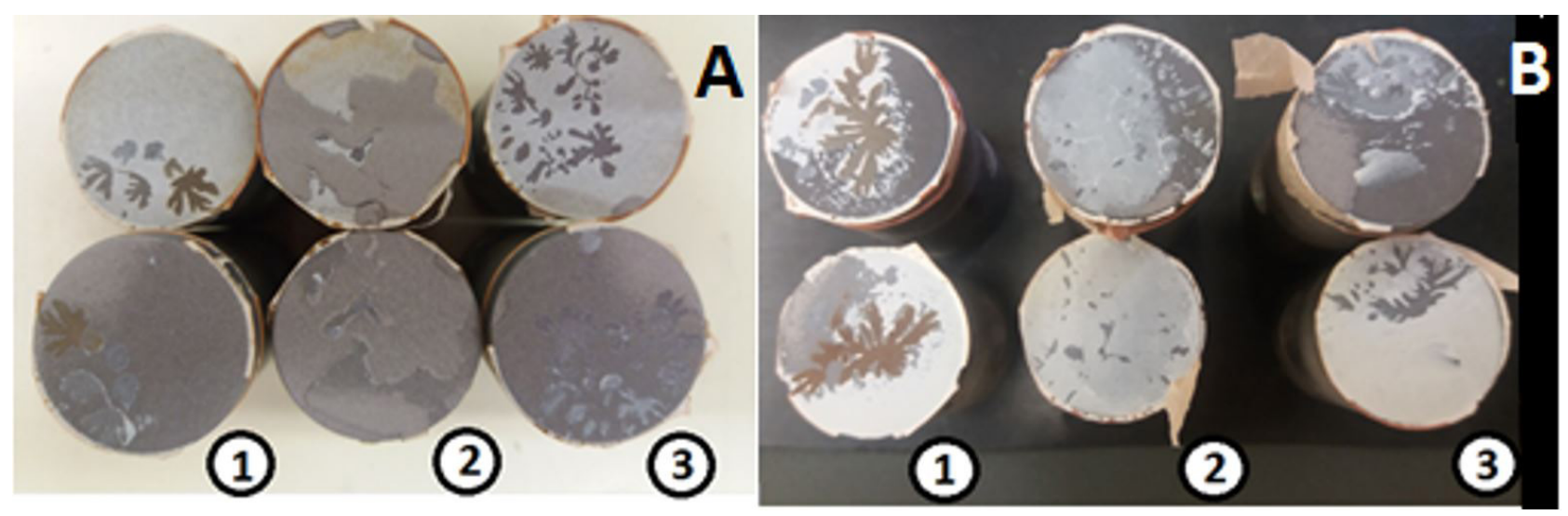

Figura 7. Interface de ruptura dos corpos de prova de tração. Imagem A do lado esquerdo, refere-se aos três pares de corpos de prova de tração ( I, 2 e 3) do processo de Plasma Spray. A Imagem B à direita mostra os três pares de corpos de prova de tração do processo de HVOF.

\section{CONCLUSÃO}

Como proposto na introdução, este estudo comparou dois processos de aspersão realizando análises mecânicas e metalográficas. Todos os parâmetros e amostras utilizadas e os processos de Plasma Spray e HVOF se mostraram adequados para realização de um comparativo qualitativo entre eles de acordo com as dimensões determinadas neste trabalho.

Este estudo é muito importante, pois fornece algumas informações valiosas aos engenheiros e tomadores de decisão sobre qual processo é apropriado para uma situação singular com base nos resultados dos testes realizados.

Em resposta à primeira pergunta, os resultados de todas as análises dos testes mecânicos e metalúrgicos, mostram uma pequena vantagem da aplicação com HVOF se comparado ao Plasma.

Em resposta à segunda pergunta, a principal vantagem do processo HVOF é que o revestimento produzido com esse processo é mais denso se comparado ao Plasma Spray, devido à alta velocidade da chama e baixas temperaturas, impedindo a oxidação excessiva da matriz metálica.
Os ganhos de conhecimento sobre as propriedades de ambas as aplicações deste revestimento em material base de titânio podem ajudar engenheiros e técnicos a decidir qual é a melhor maneira de reparar peças de titânio, que estão amplamente difundidas na indústria da aviação devido às suas propriedades exclusivas.

Pesquisas futuras com foco nos custos envolvidos em cada tipo de aplicação (Plasma Spray e HVOF) seriam uma informação valiosa. Considerando os custos e as propriedades obtidas neste estudo, os engenheiros teriam mais informações para decidir qual a melhor opção para cada situação.

\section{Agradecimentos}

Os autores agradecem ao Laboratório de Materiais da Ge Celma e ao Sistema FIRJAN, através do IST Solda, pelo suporte e infraestrutura e a todos que direta ou indiretamente auxiliaram na elaboração da pesquisa.

\section{REFERÊNCIAS}

I Tucker, R. C. An overview of alternative coatings for wear and corrosion resistance. In: Thermal Spray 1998: Meeting the Challenges of the 2 I st Century, Proceedings of the I5th International Thermal Spray Conference; Nice, France. Nice: ITSC; 1998. vol. I.

2 Huchin, J. P. The place of thermal spraying in industry today and the prospects for the future. In: Thermal Spray 1998: Meeting the Challenges of the 2 I st Century, Proceedings of the I5th International Thermal Spray Conference; Nice, France. Nice: ITSC; 1998. vol. 2.

3 Rhys-Jones TN. Thermally sprayed coating systems for surface protection and clearance control applications in aero engines. Surface and Coatings Technology. 1990;43-44:402-4I5. http://dx.doi.org/10.1016/0257-8972(90)90092-Q.

4 Lamana MS, Pukasiewicz AGM, Sampath S. Influence of cobalt content and HVOF deposition process on the cavitation erosion resistance of WC-Co coatings. Wear. 2018;398-399:209-219. http://dx.doi.org//0.1016/j. wear.2017.12.009.

5 Al-Mutairi S, Hashmi MSJ, Yilbas BS, Stokes J. Microstructural characterization of HVOF/plasma thermal spray of micro/nano WC-I $2 \%$ Co powders. Surface and Coatings Technology. 2015;264: I75-186. http://dx.doi. org/I0.1016/j.surfcoat.2014.12.050. 
6 Mishra TK, Kumar A, Sinha SK, Sharma S. Investigation of sliding wear behaviour of HVOF carbide coating. Mat. Today: Proceedings. 2018 [acesso em 3 out. 2018];5(9): 19539-19546. Disponível em: https://www.sciencedirect. com/science/article/pii/S221478531831438X

7 Chen $\mathrm{H}$, Hutchings IM. Abrasive wear resistance of plasma-sprayed tungsten carbide-cobalt coatings. Surface and Coatings Technology. 1998;107(2-3):106-I I4. http://dx.doi.org/10.1016/S0257-8972(98)0058I-7.

8 TMS Titanium. Titanium and the Aerospace Industry. Poway, CA: TMS Titanium; 2013 [acesso em I5 jun. 20I7]. Disponível em: https://tmstitanium.com/titanium-and-the-aerospace-industry/

9 Hager CH Jr, Sanders J, Sharma S, Voevodin A. Gross slip fretting wear of $\mathrm{CrCN}$, TiAIN, Ni, and CuNiln coatings on Ti6Al4V interfaces. Wear. 2007;263(I-6):430-443. http://dx.doi.org/I0.I0I6/j.wear.2006.I2.085.

10 Oerlikon Metco. Material product data sheet: tungsten carbide: 17\% cobalt agglomerated and sintered thermal spray powders. 2017.

II American Society for Testing and. ASTM E- I8: standard test methods for rockwell hardness and rockwell superficial hardness of metallic materials. Philadelphia: ASTM; 2001.

12 American Society for Testing and Materials. ASTM C-633: test method for adhesion or coeshive strength of flame sprayed coatings. West Conshohocken: ASTM; 200I.

13 American Society for Testing and Materials. ASTM E-2 109: test method for determining area percentage porosity in thermal sprayed coating. West Conshohocken: ASTM; 2007.

Recebido em: 22 Set. 2019

Aceito em: II Out. 2020 